\title{
A new approach to an influenza live vaccine: modification of the cleavage site of hemagglutinin
}

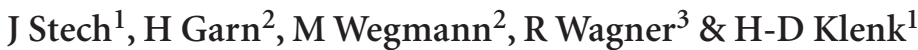

A promising approach to reduce the impact of influenza is the use of an attenuated, live virus as a vaccine. Using reverse genetics, we generated a mutant of strain A/WSN/33 with a modified cleavage site within its hemagglutinin, which depends on proteolytic activation by elastase. Unlike the wildtype, which requires trypsin, this mutant is strictly dependent on elastase. Both viruses grow equally well in cell culture. In contrast to the lethal wild-type virus, the mutant is entirely attenuated in mice. At a dose of $10^{5}$ plaque-forming units, it induced complete protection against lethal challenge. This approach allows the conversion of any epidemic strain into a genetically homologous attenuated virus.

Because of annually recurring epidemics and the enduring threat of a pandemic, influenza remains a serious public health problem despite the availability of inactivated vaccines. A live vaccine, FluMist, manufactured by MedImmune Vaccines Inc., is now commercially available in some countries. This vaccine consists of two influenza A and one influenza B reassortants of a cold-adapted master strain. These reassortants have temperature-sensitive mutations in the polymerase subunits PB2 and PB1 and the nucleoprotein genes ${ }^{1}$ and are decorated with the hemagglutinin and the neuraminidase of the circulating epidemic strain. Such a vaccine virus has the potential to provide the internal genes without temperature-sensitive mutation for reassortment with an epidemic virus and could thus give rise to a new strain with unpredictable traits ${ }^{2,3}$.

An important step in the replication cycle of the influenza virus is cleavage of the hemagglutinin by a host protease in order to gain infectivity ${ }^{4,5}$ by activating the fusion potential ${ }^{6-8}$. The cleavage site contains a conserved arginine or a stretch of basic amino acids like the highly pathogenic avian strains ${ }^{9-11}$.

Using reverse genetics, we generated in this study a mutant of strain A/WSN/33 (H1N1) with a modified hemagglutinin cleavage site that requires elastase instead of trypsin for activation. This virus is attenuated in vivo, but grows in vitro as well as the wild-type virus does, and induces a strong protection against lethal infection with the wild-type. Such an approach allows the conversion of any epidemic strain as a whole into an attenuated live vaccine virus. It is genetically homologous to the wild-type, and thus avoids the risk of generating pathogenic reassortants.

\section{RESULTS}

\section{Generation of an elastase-dependent influenza A virus}

We sought to make a virus that is not susceptible to in vivo activation at the basic hemagglutinin cleavage site but that can be activated in vitro by a protease not available under natural conditions. Wild-type hemagglutinin is cleaved by trypsin-like serine proteases, and cleavage generates two fragments, HA1 and HA2. We had to consider two requirements for the choice of the cleavage motif of such a protease. First, the glycine had to be retained at position P1 because this amino acid is essential at the amino terminus of the HA2 fragment for induction of fusion and cannot be replaced without compromising or possibly abolishing fusion ${ }^{10,12-14}$. Second, to reduce the probability of reversion, we had to select an amino acid whose codon differs by two nucleotides from any arginine or lysine codon ${ }^{15,16}$. We therefore exchanged the A at position 1,059 and the $\mathrm{G}$ at position 1,060 of the A/WSN/33 hemagglutinin with $\mathrm{G}$ and $\mathrm{T}$, respectively, replacing Arg343 with valine and generating a cleavage site for porcine pancreatic elastase $\mathrm{e}^{17}$ (Fig. 1). Using a previously established reverse genetics system $^{18}$, we generated recombinant viruses containing either authentic (WSNwt) or mutated (which can be cleaved by elastase; WSNE) hemagglutinin. We then sequenced the gene encoding the HAl peptides of WSNwt and WSN-E and found no nucleotide differences (data not shown).

\section{WSN-E is activated by elastase exclusively}

Multicycle replication and thus plaque formation require proteolytic activation ${ }^{4}$. Therefore, we performed plaque assays on Madin-Darby canine kidney (MDCK) cells in the presence of either trypsin or elastase in the plaque overlay or in the absence of an exogenous protease for control. With WSNwt, clear plaques were only visible in the presence of trypsin. In contrast, WSN-E was exclusively activated by elastase

\footnotetext{
${ }^{1}$ Institut fuer Virologie, Klinikum der Philipps-Universitaet Marburg, Postfach 2360, 35011 Marburg, Germany. ${ }^{2}$ Institut fuer Klinische Chemie und Molekulare Diagnostik, Biomedizinisches Forschungszentrum (BMFZ) Klinikum der Philipps-Universitaet Marburg, Hans-Meerwein-Straße, 35033 Marburg, Germany.

${ }^{3}$ Paul-Ehrlich-Institut, Bundesamt fuer Sera und Impfstoffe, Paul-Ehrlich-Str. 51-59, 63225 Langen, Germany. Correspondence should be addressed to J.S. (stech@med.uni-marburg.de).
} 


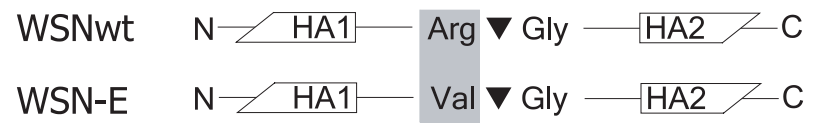

$$
\begin{array}{ll}
\text { WSNwt } & 5^{\prime} \mathrm{HA} \text { AG A GGT HA2 } 3^{\prime} \\
\text { WSN-E } & 5^{\prime} \succ \text { HA1 GT A GGT HA2 }{ }^{\prime}
\end{array}
$$

Figure 1 Cleavage sites of WSNwt and WSN-E. The monobasic cleavage site of the WSNwt hemagglutinin contains an arginine, which has been replaced by a valine in the case of WSN-E.

(Fig. 2a), even if only one wash with PBS was done before inoculation. These results demonstrate that neither plasmin shown to activate WSNwt ${ }^{5,19}$ nor another protease provided by MDCK cells or fetal calf serum is able to cleave WSN-E hemagglutinin. We analyzed the dependence of the hemagglutinin cleavage in WSNwt on trypsin and in WSN-E on elastase by western blotting. The results show that the hemagglutinin of WSN-E is cleaved by elastase, but is resistant to trypsin (Fig. 2b). The weak HAl band, which appeared after incubation of WSNwt with elastase, can be attributed to incomplete cleavage between amino acids glycine and leucine ${ }^{17}$. This cleavage does not cause proteolytic activation, as shown by the plaque assay (Fig. 2a). In the presence of the appropriate protease, both viruses grew to similar titers, although kinetics were somewhat slower with WSN-E (Fig. 2c).

\section{WSN-E is attenuated in mice, whereas WSNwt is lethal}

To analyze WSN-E in vivo, we infected mice with $10^{6}$ plaque-forming units (p.f.u.) of either WSNwt or WSN-E and observed them for $14 \mathrm{~d}$. When infected with wild-type virus, mice $(n=3)$ showed signs of disease and died on day 7. In the case of WSN-E, all mice $(n=4)$ survived (Fig. 3a) without weight loss (Fig. 3b) or any visible symptoms of disease.

\section{WSN-E undergoes restricted replication in the lung}

To compare the pathogenic traits of both viruses in vivo, we inoculated mice with either PBS, $10^{6}$ p.f.u. WSNwt or $10^{6}$ p.f.u. WSN-E. After $12 \mathrm{~h}, 1 \mathrm{~d}$ and $3 \mathrm{~d}$, we removed lung, brain and heart. Beginning with undiluted organ homogenate, we performed plaque titrations with WSNwt in the presence of trypsin and with WSN-E in the presence of elastase. We found WSNwt in the lungs up to day 3, showing a rise in titer. Wild-type virus was also detectable in brain and heart. Mice inoculated with WSN-E showed different characteristics. We did not find WSN-E in brain or heart at any time point analyzed. In the lung, viral titers stagnated from $12 \mathrm{~h}$ to $1 \mathrm{~d}$ after inoculation. On day 3 , we could not detect any WSN-E virus (Fig. 3c-e). The lung titer of WSN-E at $12 \mathrm{~h}$ was approximately $1.4 \times 10^{6} \mathrm{p}$.f.u. per mouse lung, which is close to the inoculum dose. For WSNwt, the result was considerably different. The lung titer at $12 \mathrm{~h}$ was approximately $2.2 \times 10^{8}$ p.f.u. per mouse lung, showing that WSNwt readily multiplies in the lung. In contrast, replication of WSN-E is abortive because it is restricted in vivo to one replication cycle because of the absence of the appropriate protease.

Figure 2 In vitro properties of WSNwt and WSN-E. Plaque assay (a) and western blot (b) of WSNwt and WSN-E in the absence of an exogenous protease, in the presence of trypsin or elastase. Growth curves (c) of WSNwt (circles) in the presence of trypsin and WSN-E (squares) in the presence of elastase.

\section{WSN-E is found only after first passage in mouse lung}

To check for the emergence of revertants, we carried out sequential lung passages in mice. At the first passage, we inoculated the animals with $10^{6}$ p.f.u. WSN-E. For the subsequent infections, we used $50 \mu \mathrm{l}$ lung homogenate from the previous passage. During five lung passages of either $1 \mathrm{~d}$ or $3 \mathrm{~d}$ duration, the mice remained unaffected. We determined the virus titers by plaque assay in the presence of elastase. Because WSNwt is activated by plasmin ${ }^{5,19}$, this virus can be detected in absence of trypsin if only one wash is performed before inoculation. We did not detect any virus, even in undiluted lung homogenate obtained from the second to the fifth of the 1-d passages and from the first to the fifth of the 3 -d passages (data not shown). This shows the absence of WSN-E and of revertants.

\section{Reversion of WSN-E in vitro}

After the first passage in mouse lung, the entire amount of WSN-E was in the range of $10^{5}-10^{6}$ p.f.u.. Such virus populations are too small for the generation of double-point revertants, having an equilibrium frequency of approximately $10^{-5}$ to $10^{-8}$ (ref. 20). Therefore, we passaged the elastase-dependent WSN-E on MDCK cells in the presence

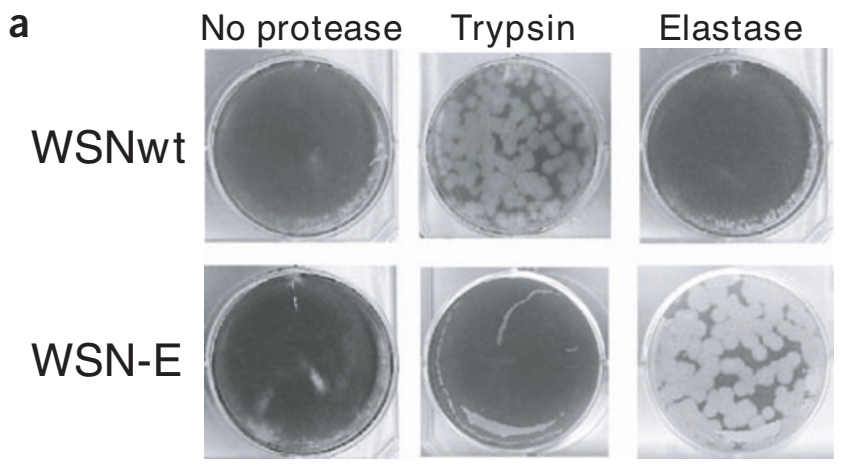

b

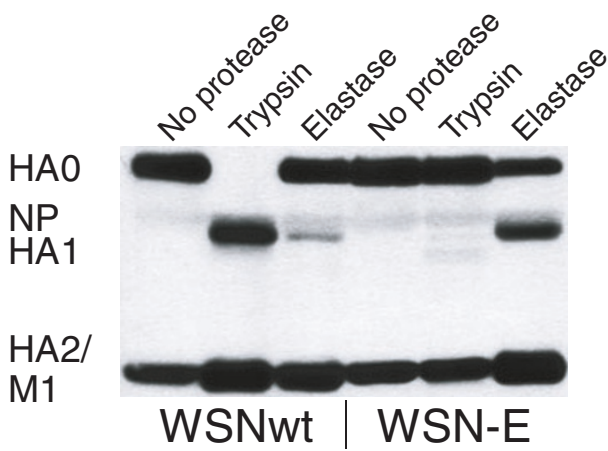

C

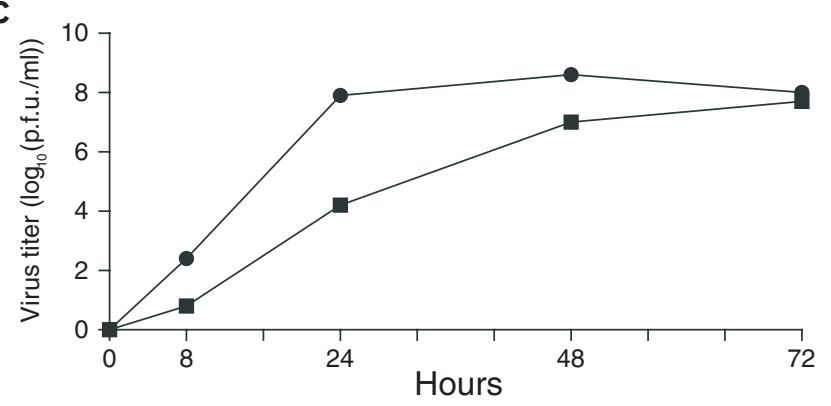


Figure 3 Pathogenicity and organ tropism of WSNwt and WSN-E in mice. Survival rates (a) and average weight loss (b) after infection with WSNwt (circles) or WSN-E (squares). Plaque titers from entire lungs (c), brains (d) and hearts (e) removed at $12 \mathrm{~h}, 1 \mathrm{~d}$ or $3 \mathrm{~d}$.

of trypsin, beginning with inocula of $10^{8}, 10^{7}$ or $10^{6}$ p.f.u. each in ten parallel cell cultures. From all $10^{8}$ p.f.u. inocula and from six out of ten $10^{7}$ p.f.u. inocula, we found trypsindependent virus with lysine at its cleavage site. We could not obtain any revertants from inocula of $10^{6}$ p.f.u.. Therefore, the reversion frequency within the WSN-E stock is approximately $10^{-7}$. The low reversion rate and the small viral loads of WSN-E in the mouse lung explain the absence of revertants during mouse passages. Another reason for the genetic stability of WSN-E in vivo is the restriction to one replication cycle because of the absence of the appropriate protease.

\section{Protection against lethal challenge}

To investigate the potential of WSN-E to serve as a live vaccine, we immunized five groups of mice with WSN-E: four groups received live virus at dosages of $10^{3}(n=6), 10^{4}(n=6)$, $10^{5}(n=6)$ or $10^{6}$ p.f.u. $(n=5)$; one group received formalin-inactivated virus $(n=4)$. An additional group of nonimmunized mice $(n=6)$ served as a positive control during challenge. The animals tolerated the immunization without any signs of illness, as in the previously described survival experiment. Four weeks later, we challenged the mice with $10^{6}$ p.f.u. WSNwt and monitored survival and

weight loss (Fig. 4a,b). The challenge was lethal for both groups vaccinated with formalin-inactivated virus or $10^{3}$ p.f.u. WSN-E, as well as for the nonimmunized control animals. From the mice immunized with $10^{4}$ p.f.u. WSN-E, four of six mice survived and partially recovered from disease. Although some animals from the group vaccinated with $10^{5}$ p.f.u. WSN-E developed temporary weight loss and milder disease symptoms, they eventually recovered. All animals vaccinated with the highest dose of $10^{6}$ WSN-E survived the challenge and did not develop any weight loss or other visible symptoms of illness. We removed the lungs of two mice per group for plaque assay $3 \mathrm{~d}$ after challenge (Fig. 4c). Notably, after vaccination with $10^{6}$ p.f.u. WSN$\mathrm{E}$, no plaques were seen, even in undiluted lung homogenates. This contrasts considerably with the plaque titers of the other groups. The challenge experiment indicates that the degree of protection against disease increases with the immunization dose. Additionally, the failure of the formalin-inactivated virus to prevent death shows that WSNE must replicate to induce protection. Taken together, these results show that WSN-E is an attenuated virus that is able to prevent lethal influenza virus infection.

\section{Strong serum and mucosal antibody response}

We immunized mice with $10^{3}(n=6, n=5), 10^{4}(n=5, n=4), 10^{5}$ $(n=5, n=6)$ or $10^{6}$ p.f.u. $(n=4, n=6)$ WSN-E, $10^{6}$ p.f.u. formalin-
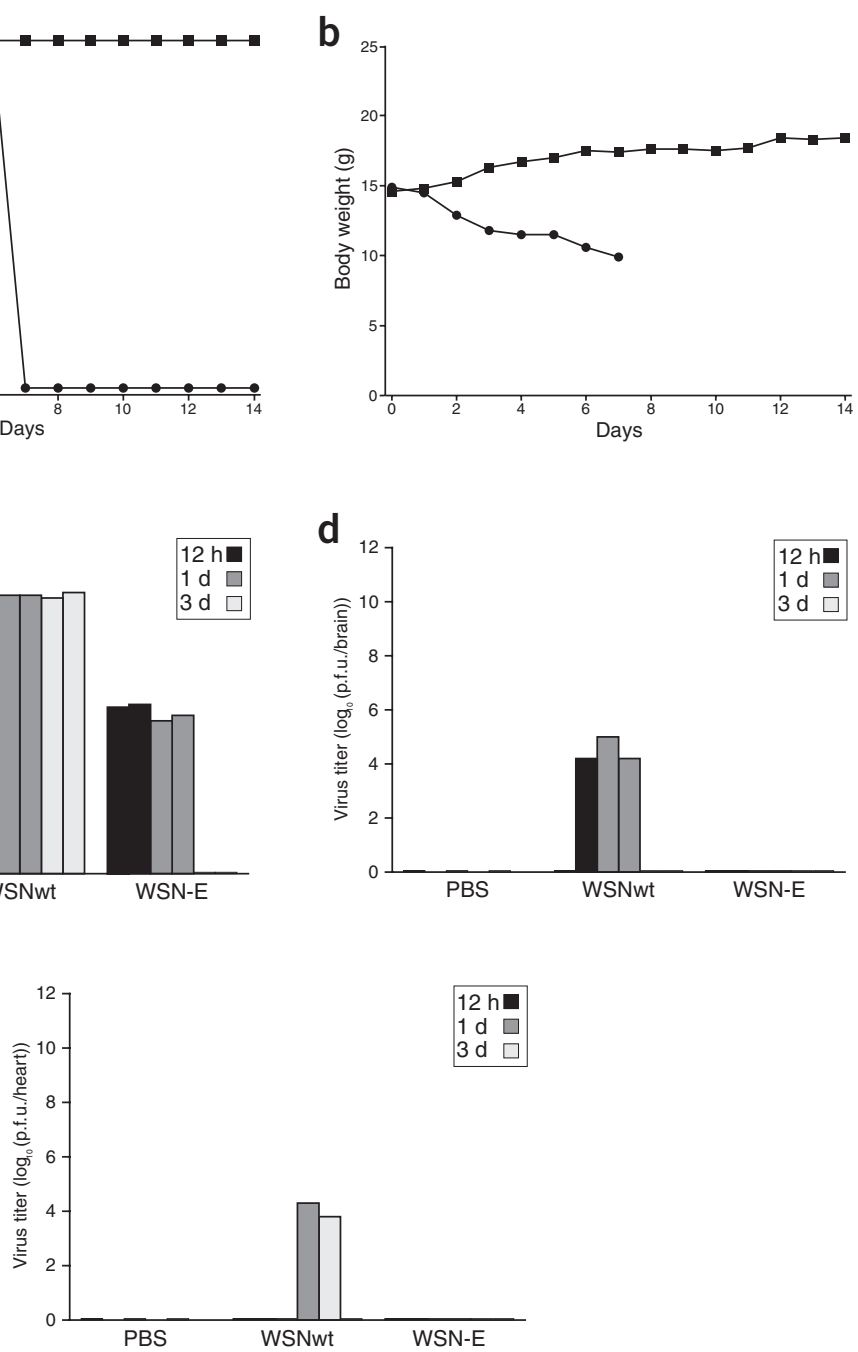

inactivated WSN-E ( $n=5, n=4)$, WSNwt at a nonlethal dosage of $10^{3}$ p.f.u. $(n=5, n=7)$, or with PBS $(n=6, n=6)$. Mice were killed 4 weeks later. We challenged a subgroup of each treatment cohort with $10^{6}$ p.f.u. WSNwt $3 \mathrm{~d}$ before analysis. To investigate the virus-specific antibody response, we determined the IgG titers of sera and IgA titers of bronchoalveolar (BAL) fluid and nasal wash samples by ELISA. Additionally, we performed serum hemagglutination inhibition tests. Mice that received $10^{3}$ p.f.u. WSN-E, formalin-inactivated WSN-E or PBS did not show any antibody response before or after challenge. By contrast, the groups immunized with $10^{4}, 10^{5}$ or $10^{6}$ p.f.u. WSN-E showed substantial levels of virus-specific IgG and hemagglutinin inhibition titers in serum as well as IgA titers in BAL and nasal wash, which increased with the immunization dose (Fig. 5a-d). We achieved the highest antibody titers with $10^{3}$ p.f.u. WSNwt. With $10^{6}$ p.f.u. WSN-E, the hemagglutinin inhibition titer before challenge was 1:40 (Fig. 5b). Compared with nonchallenged animals, challenged mice of the WSN-E $10^{6}$ p.f.u. group showed elevated antibody titers (Fig. 5a-d), especially in the nasal wash (Fig. 5d). This may be attributed both to antigenic boost and induction of a fast and effective memory immune response.

\section{DISCUSSION}

Our goal was to generate influenza A virus with an atypical hemagglutinin cleavage site that is resistant to activation during natural 


\section{TECHNICAL REPORTS}

a

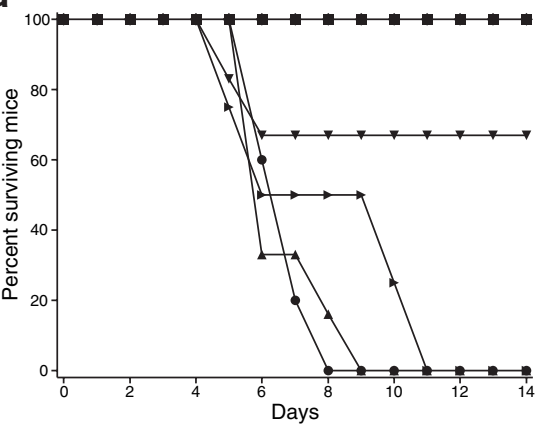

b

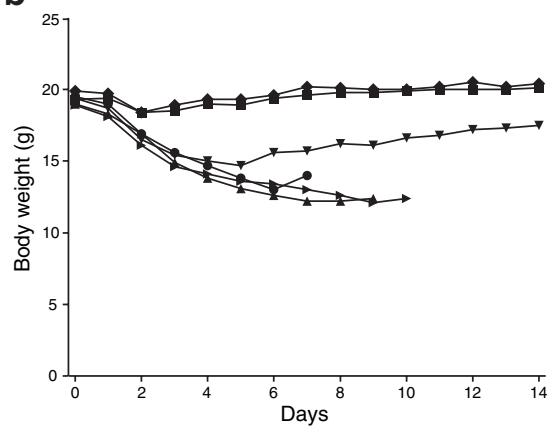

C

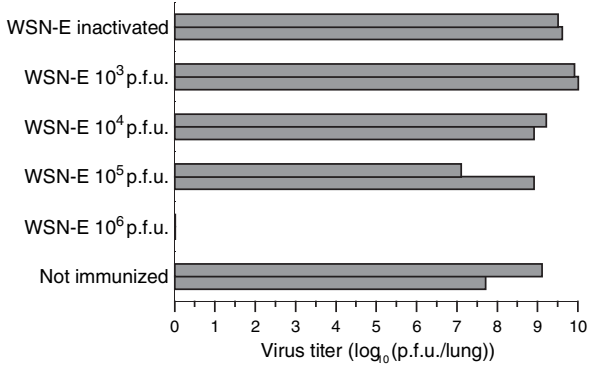

Figure 4 Protection against lethal challenge with WSNwt. Survival rates (a) and average weight loss (b) of challenged mice immunized with formalininactivated WSN-E (right-facing triangles), $10^{3}$ p.f.u. WSN-E (triangles), $10^{4}$ p.f.u. WSN-E (inverted triangles), $10^{5}$ p.f.u. WSN-E (diamonds) or $10^{6}$ p.f.u. WSN-E (squares) and nonimmunized (circles). Lung plaque titers (c) from the third day.

infection but can readily be activated under in vitro conditions. We accomplished this by replacing the original trypsin-specific cleavage site Arg-Gly with the elastase-sensitive site Val-Gly. Elastase mutants have previously been obtained after conventional cell-culture passages in the presence of this enzyme ${ }^{21}$. This study shows, however, that by reverse genetics generation of such mutants has become a fast and reproducible procedure suitable for routine production. WSNwt and the elastase-substituted WSN-E grew to similar titers in cell culture. In mouse lung, WSN-E was present only temporarily and did not cause any disease. But after infection with wildtype virus, we observed much higher lung titers, spread of virus to other organs and $100 \%$ lethality. Thus, the cleavage site mutant proved to be equivalent to wild-type virus regarding growth rates in vitro, but was completely attenuated in vivo. Because of these properties, WSN-E is a promising candidate for a live vaccine.

Unlike WSNwt, WSN-E titers in the mouse lung hardly exceeded the intranasal inoculum, indicating that replication was restricted to very few cycles. Accordingly, mice had to be vaccinated with relatively high doses of WSN-E ( $10^{6}$ or $10^{5}$ p.f.u.) for immunity against lethal challenge. But when inoculated with $10^{6}$ p.f.u. inactivated WSN-E, mice neither survived a challenge with WSNwt nor developed a detectable antibody response. It may be argued that the formalin inactivation was too harsh, but the protein amount used was approximately $80 \mathrm{ng}$ per immunization dose. Moreover, we prepared the formalin-

Figure 5 Serum and mucosal antibody response. Serum IgG titers (a), serum hemagglutination inhibition (HI) titers (b), bronchoalveolar lavage (BAL) IgA titers (c), and nasal wash IgA titers (d) of nonchallenged (black bars) and challenged (gray bars) mice immunized with PBS, $10^{3}$ p.f.u. WSN-E, $10^{4}$ p.f.u. WSN-E, $10^{5}$ p.f.u. WSN-E, $10^{6}$ p.f.u. WSN-E, $10^{6}$ p.f.u. formalininactivated WSN-E and $10^{3}$ p.f.u. WSNwt. inactivated WSN-E from the same nonconcentrated virus stock used for the live immunization and inoculated it intranasally only once (like the live WSN-E). Such inactivated vaccines are made from concentrated virus and usually administered three times to the mice ${ }^{22}$. The failure of immunization with formalin-inactivated virus showed that WSN-E replication was required for protection.

One intranasal inoculation of WSN-E induced a substantial, dosedependent local and systemic immune response despite very limited presence in lung. A dosage of $10^{5}$ or $10^{6}$ p.f.u. induced substantial a
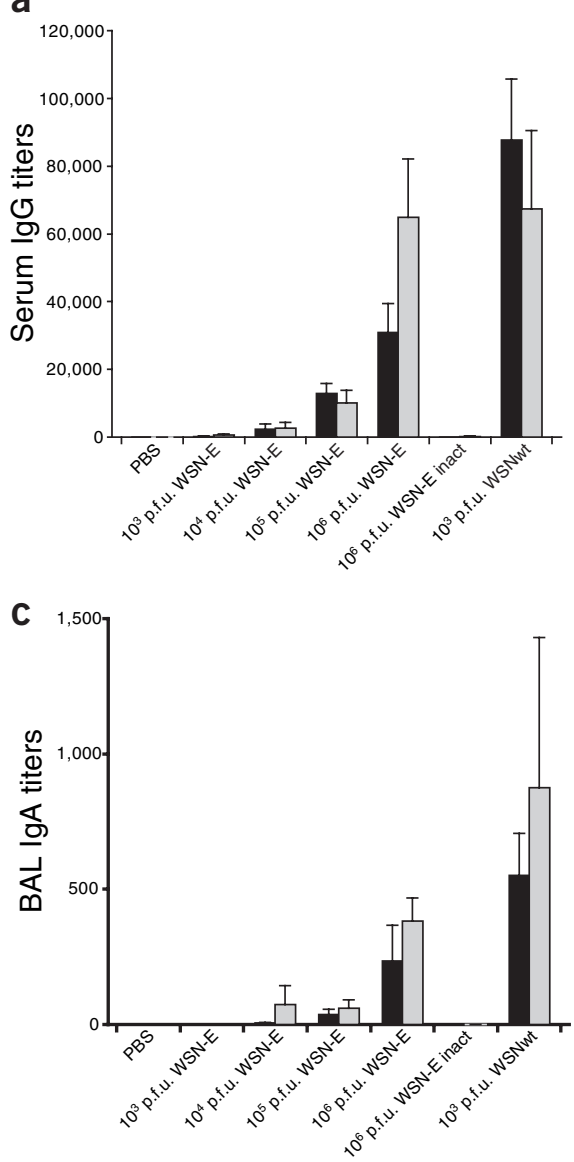

b
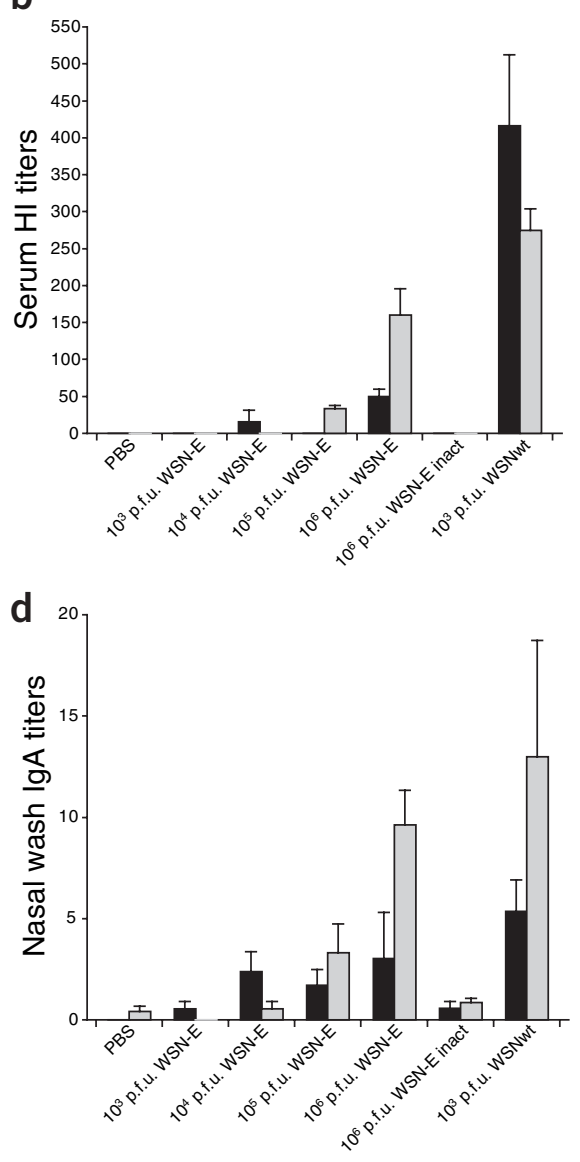
hemagglutination inhibition titers, serum IgG and mucosal IgA titers. They were lower than those induced by $10^{3}$ p.f.u. WSNwt because its longer replication enables antigenic stimulation. But the challenged animals showed almost comparable systemic and mucosal immunoglobulin titers when immunized with $10^{6}$ p.f.u. WSN-E. This indicates that a notable immunological memory had already been induced in these animals.

A frequent objection against the use of live influenza virus vaccines is the possibility of reversion to pathogenicity. With WSN-E, we ๖ obtained no evidence for revertants after five passages in mice. This may be explained in part by the highly restricted replication of this virus in the lung which results in small viral loads. The absence of appropriate proteases for WSN-E in vivo allows only one (or very few) replication cycle(s) leading to self-limiting replication. This is the main difference from other attenuated viruses undergoing many replication cycles in vivo. For cold-adapted viruses, a duration of viral shedding up to $11 \mathrm{~d}$ in susceptible humans was reported ${ }^{23}$. An important advantage of the short self-limiting replication is the decreased probability of any reversion, including the cleavage site motif itself and other attenuating mutations. Another factor contributing to the absence of reversion in vivo is the double mutation within the cleavage site. For back-mutation, two nucleotides at once have to be replaced because suppressor mutants outside of the cleavage region seem to be impossible. This 음 explains the low reversion frequency in cell culture. In hen eggs, a factor X-like protease is present ${ }^{24}$, which should cause a considerably higher proportion of revertants. Therefore, eggs might not be suitable for vaccine production. In cell culture, the substitution of trypsin by elastase for propagation of WSN-E leads both to positive selection for elastase-dependent virus and to negative selection against revertants.

Live influenza vaccines presently approved for human application are reassortants generated by coinfection of a cold-adapted temperature-sensitive master strain from which the six segments coding for the internal virion components are derived and the circulating strain which provides the hemagglutinin and neuraminidase genes ${ }^{25,26}$. The faster generation of such reassortants by reverse genetics entirely from plasmids ${ }^{1,18,27}$ is feasible as well. These live vaccines are well tolerated and effective ${ }^{28-30}$. But such an attenuated virus may give rise to a new viral strain with unpredictable traits by exchanging the internal genes, especially those without temperature-sensitive mutations (genes encoding the polymerase subunit PA, matrix and nonstructural proteins $)^{1}$, with the circulating strain. Experimental evidence for generation of a pathogenic virus from reassortment of two apathogenic strains has indeed been obtained ${ }^{2,3}$. Such a scenario is avoided when a cleavage-site mutant is used that contains all eight genes of the circulating strain. A cleavage site mutant would deliver all antigens identical to the circulating strain and, therefore, be the most authentic vaccine. The possible advantage of this feature is indicated by studies showing that the internal influenza virion components prime a helper response cooperating in the antibody response against the hemagglu$\operatorname{tinin}^{31,32}$. The immunogenic relevance of the internal components is also underlined by the observation that live virus, and to some extent inactivated whole-virus vaccine, can induce heterotypic protection in contrast to subunit vaccines ${ }^{33}$. Furthermore, it has been reported ${ }^{34}$ that mice could be protected successfully from lethal infection with $\mathrm{A} /$ HongKong/156/97 (H5N1) by prior immunization with the A/Quail/ HongKong/G1/97 (H9N2) isolate, which harbors internal genes 98\% homologous to the $\mathrm{H} 5 \mathrm{~N} 1$ isolate.

In vaccine production, some circulating strains may grow to inadequate titers. Therefore, the propagation of such a seed virus rescued from genes of the epidemic strain may be delayed unpredictably. A solution would be to adapt an epidemic strain to cell lines suitable for vaccine production and to use its internal genes as a backbone each year. The internal genes evolve considerably more slowly than the surface glycoproteins ${ }^{35}$. Thereby, both high-growth properties and sufficient antigenic homology of the internal viral proteins can be provided. Moreover, this backbone can carry additional attenuating mutations.

To show that our approach is generalizable to highly pathogenic influenza strains, we recently generated an elastase-dependent mutant of the strain SC35M. This virus is an H7N7 isolate, carries a polybasic cleavage site and is highly pathogenic both for chickens and mice ${ }^{36}$. Like WSN-E, the SC35M mutant is strictly dependent on elastase and grows to similar titers in cell culture like the wild-type (G. Mehmetoglu \& J.S., unpublished data).

Because proteolytic activation is essential for the replication of each influenza virus, the conversion of any epidemic strain or of viruses with pandemic potential, such as highly pathogenic H5N1 strains, into a live vaccine by altering the cleavage site is possible. Major assets of cleavage site mutants are antigenic identity to the parent strain, nonexisting risk of generating new pathogenic reassortants, complete attenuation in vivo and in vitro growth equivalent to wild-type. Such an attenuated virus is an ideal candidate for a live vaccine.

\section{METHODS}

Cells. We cultured 293T human embryonic kidney cells in Dulbecco minimal essential medium containing 10\% FCS and MDCK cells in minimal essential medium supplemented with 10\% FCS.

Recombinant viruses. For generation of the recombinant influenza viruses, we used a previously described reverse genetics system ${ }^{18}$. This system is based on transfection of eight plasmids encoding the genes of strain A/WSN/33 (H1N1) under control of the human RNA1 promoter and mouse RNA polymerase I terminator and a truncated RNA polymerase II (immediate-early CMV) promoter. To rescue WSNwt, we used the eight original plasmids ${ }^{18}$ mentioned above. For WSN-E, we mutagenized the hemagglutinin-encoding plasmid pHW184-HA to replace Arg343 with valine. We changed the nucleotides corresponding to positions 1,059 and 1,060 in the A/WSN/33 hemagglutinin gene from AG to GT by using the Quikchange Kit (Stratagene). The primer sequences are: $5^{\prime}$ CCCATCCATTCAATACGTAGGTCTATTTGGAGCCA-3' and 5'-TGGCTCC AAATAGACCTACGTATTGAATGGATGGG-3'.

Virus propagation. After rescue, we plaque-purified and grew both WSNwt and WSN-E on MDCK cells in minimal essential medium containing $0.2 \%$ bovine serum albumin. For propagation of WSNwt and WSN-E, we used $0.5 \mu \mathrm{g} / \mathrm{ml}$ $\mathrm{N}$-tosyl-L-phenylalanine chloromethyl ketone (TPCK)-treated trypsin (Sigma) and $5 \mu \mathrm{g} / \mathrm{ml}$ porcine pancreatic elastase (Serva Electrophoresis $\mathrm{GmbH}$ ), respectively. For growth curves, we inoculated MDCK cells with virus at multiplicity of infection of $3 \times 10^{-2}$.

Plaque assay. We performed the plaque assays on MDCK cell monolayers essentially as described ${ }^{37}$. Before inoculation, we washed the cells five times with PBS. For passage experiments, we performed only one wash. For WSN-E, we used elastase instead of TPCK-treated trypsin in the plaque overlay.

Passage experiments in vitro. We grew WSN-E in 10 separate flasks $\left(165 \mathrm{~cm}^{2}\right)$ on MDCK cells in minimal essential medium containing $0.2 \%$ bovine serum albumin in the presence of $0.5 \mu \mathrm{g} / \mathrm{ml}$ TPCK-treated trypsin. The next day, we transferred entire supernatants to ten flasks with fresh MDCK cells. After $2 \mathrm{~d}$, we transferred half of each volume of supernatant into ten fresh flasks. We performed another two passages of $2 \mathrm{~d}$ unless a cytopathic effect occurred. From the supernatants of the last passage, we performed RT-PCR for sequencing of the cleavage site of the hemagglutinin.

Western blot. We washed confluent MDCK monolayers, grown in dishes $6 \mathrm{~cm}$ in diameter, five times with PBS and infected them with WSNwt or WSN-E at multiplicity of infection of 10 . We incubated both viruses in the presence of 


\section{TECHNICAL REPORTS}

either TPCK-treated trypsin, elastase or no protease for $16 \mathrm{~h}$ in minimal essential medium containing $0.2 \%$ bovine serum albumin. After SDS-PAGE (10\%) from pelleted supernatants, we used a polyclonal rabbit antiserum to A/WSN/33 and an rabbit-specific swine monoclonal antibody, conjugated with horseradish peroxidase (Dako) as secondary antibody (each $1 \mathrm{~h}$ at $22^{\circ} \mathrm{C}, 1: 2,000$ ) followed by chemiluminescence (Supersignal West Pico Chemiluminescent Substrate kit from Pierce).

Formalin inactivation. We treated WSN-E at a concentration of $5 \times 10^{7}$ p.f.u./ $\mathrm{ml}$ (equivalent to $10^{6}$ p.f.u. in $20 \mu \mathrm{l}$ used for intranasal inoculation) with $0.1 \%$ formaldehyde and incubated it for one week at $4{ }^{\circ} \mathrm{C}$. By plaque assay, we confirmed the absence of live virus. The hemagglutination titer of formalin-inactivated WSN-E was 1:64, that of live WSN-E 1:128. The protein content of $10^{6}$ p.f.u. WSN-E virus stock was approximately $80 \mathrm{ng}$.

Animal experiments. The animal experiments were performed according to the guidelines of the German animal protection law. All animal protocols were approved by the relevant German authority, the Regierungspräsidium Gießen. We intranasally inoculated 4-week-old female Balb/C mice (Charles River) with $20 \mu \mathrm{l}$ virus suspension under anesthesia after an intramuscular injection of ketamine hydrochloride at a dosage of $200 \mathrm{mg} / \mathrm{kg}$. At respective time points, mice were killed by cervical dislocation and entire organs removed. After homogenization in $1 \mathrm{ml}$ PBS, we determined the organ titers by plaque assay in the presence of the appropriate protease in the overlay (inoculum size $333 \mu \mathrm{l}$ ).

Antibody assays. We detected serum IgG antibodies and IgA antibodies in bronchoalveolar lavage fluid and nasal washes by ELISA. According to previously described methods ${ }^{38}$, we obtained BAL fluid and nasal wash samples. First, we coated Maxisorp 96-well plates (Nunc) with total WSNwt virus protein at $4{ }^{\circ} \mathrm{C}$ overnight. Then, we detected the bound antibodies by mouse-specific IgG or IgA labeled with horseradish peroxidase (BD Pharmingen) and BM Blue POD substrate (Roche Diagnostics). Finally, we expressed the titers as reciprocal of the dilution that yielded an optical density of 0.1 . We performed the hemagglutination inhibition test ${ }^{39}$ after incubation overnight with receptor-destroying enzyme (Dade-Behring) at $37^{\circ} \mathrm{C}$ and heat inactivation at $56^{\circ} \mathrm{C}$ for $30 \mathrm{~min}$ using chicken erythrocytes and 4 hemagglutinating units of WSNwt.

Accession number. The GenBank accession number for the A/WSN/33 hemagglutinin precursor is J02176 and for TPA: Mus musculus ribosomal DNA, complete repeating unit it is BK000964.

\section{ACKNOWLEDGMENTS}

This work was supported by grants from the Deutsche Forschungsgemeinschaft (SFB 593) and from the Fonds der Chemischen Industrie. We are grateful to E. Hoffmann and R. G. Webster for providing us the plasmids of the reverse genetics system. We thank C. Scholtissek for critical reading of the manuscript. For technical expertise and support, we are indebted to A. Herwig, G. Schemken, S. Berthel, U. Lanzinger, A. Spies and A. Wensing.

\section{COMPETING INTERESTS STATEMENT}

The authors declare competing financial interests (see the Nature Medicine website for details).

Received 15 March; accepted 4 May 2005

Published online at http://www.nature.com/naturemedicine/

1. Jin, $\mathrm{H}$. et al. Multiple amino acid residues confer temperature sensitivity to human influenza virus vaccine strains (FluMist) derived from cold-adapted A/Ann Arbor/6/60. Virology 306, 18-24 (2003)

2. Scholtissek, C., Vallbracht, A., Flehmig, B. \& Rott, R. Correlation of pathogenicity and gene constellation of influenza A viruses. II. Highly neurovirulent recombinants derived from non-neurovirulent or weakly neurovirulent parent virus strains. Virology 95, 492-500 (1979).

3. Yamnikova, S.S. et al. A reassortant H1N1 influenza A virus caused fatal epizootics among camels in Mongolia. Virology 197, 558-563 (1993).

4. Klenk, H-D., Rott, R., Orlich, M. \& Blodorn, J. Activation of influenza A viruses by trypsin treatment. Virology 68, 426-439 (1975).

5. Lazarowitz, S.G. \& Choppin, P.W. Enhancement of the infectivity of influenza A and $B$ viruses by proteolytic cleavage of the hemagglutinin polypeptide. Virology 68 , 440-454 (1975)

6. Maeda, T. \& Ohnishi, S. Activation of influenza virus by acidic media causes hemolysis and fusion of erythrocytes. FEBS Lett. 122, 283-287 (1980).
7. Huang, R.T., Wahn, K., Klenk, H-D. \& Rott, R. Fusion between cell membranes and liposomes containing the glycoprotein of influenza virus. Virology 104, 294-302 (1980).

8. White, J., Matlin, K. \& Helenius, A. Cell fusion by Semliki Forest, influenza, and vesicular stomatitis viruses. J. Cell Biol. 89, 674-679 (1981).

9. Bosch, F.X., Garten, W., Klenk, H-D. \& Rott, R. Proteolytic cleavage of influenza virus hemagglutinins: primary structure of the connecting peptide between $\mathrm{HAl}$ and HA2 determines proteolytic cleavability and pathogenicity of Avian influenza viruses. Virology 113, 725-735 (1981).

10. Garten, W., Bosch, F.X., Linder, D., Rott, R. \& Klenk, H-D. Proteolytic activation of the influenza virus hemagglutinin: The structure of the cleavage site and the enzymes involved in cleavage. Virology 115, 361-374 (1981)

11. Suarez, D.L. et al. Comparisons of highly virulent H5N1 influenza A viruses isolated from humans and chickens from Hong Kong. J. Virol. 72, 6678-6688 (1998).

12. Cross, K.J., Wharton, S.A., Skehel, J.J., Wiley, D.C. \& Steinhauer, D.A. Studies on influenza haemagglutinin fusion peptide mutants generated by reverse genetics. EMBO J. 20, 4432-4442 (2001).

13. Qiao, H., Armstrong, R.T., Melikyan, G.B., Cohen, F.S. \& White, J.M. A specific point mutant at position 1 of the influenza hemagglutinin fusion peptide displays a hemifusion phenotype. Mol. Biol. Cell 10, 2759-2769 (1999).

14. Steinhauer, D.A., Wharton, S.A., Skehel, J.J. \& Wiley, D.C. Studies of the membrane fusion activities of fusion peptide mutants of influenza virus hemagglutinin. J. Virol. 69, 6643-6651 (1995).

15. Gunther, I., Glatthaar, B., Doller, G. \& Garten, W.A. H1 hemagglutinin of a human influenza A virus with a carbohydrate-modulated receptor binding site and an unusual cleavage site. Virus Res. 27, 147-160 (1993).

16. Kawaoka, Y., Yamnikova, S., Chambers, T.M., Lvov, D.K. \& Webster, R.G. Molecular characterization of a new hemagglutinin, subtype $\mathrm{H} 14$, of influenza A virus. Virology 179, 759-767 (1990).

17. Mecham, R.P. et al. Elastin degradation by matrix metalloproteinases. Cleavage site specificity and mechanisms of elastolysis. J. Biol. Chem. 272, 18071-18076 (1997).

18. Hoffmann, E., Neumann, G., Kawaoka, Y., Hobom, G. \& Webster, R.G.A. DNA transfection system for generation of influenza A virus from eight plasmids. Proc. Natl Acad. Sci. USA 97, 6108-6113 (2000).

19. Lazarowitz, S.G., Goldberg, A.R. \& Choppin, P.W. Proteolytic cleavage by plasmin of the HA polypeptide of influenza virus: host cell activation of serum plasminogen. Virology 56, 172-180 (1973).

20. Ribeiro, R.M., Bonhoeffer, S. \& Nowak, M.A. The frequency of resistant mutant virus before antiviral therapy. AIDS 12, 461-465 (1998).

21. Orlich, M., Linder, D. \& Rott, R. Trypsin-resistant protease activation mutants of an influenza virus. J. Gen. Virol. 76, 625-633 (1995)

22. Takada, A., Matsushita, S., Ninomiya, A., Kawaoka, Y. \& Kida, H. Intranasal immunization with formalin-inactivated virus vaccine induces a broad spectrum of heterosubtypic immunity against influenza A virus infection in mice. Vaccine 21, 3212-3218 (2003).

23. Wright, P.F., Sell, S.H., Shinozaki, T., Thompson, J. \& Karzon, D.T. Safety and antigenicity of influenza A/Hong Kong/68-ts-1 (E) (H3N2). J. Pediatr. 87, 1109-1116 (1975).

24. Gotoh, B. et al. An endoprotease homologous to the blood clotting factor $X$ as a determinant of viral tropism in chick embryo. EMBO J. 9, 4189-4195 (1990).

25. Maassab, H.F. Adaptation and growth characteristics of influenza virus at 25 degrees C. Nature 213, 612-614 (1967)

26. Murphy, B.R. et al. Cold-adapted variants of influenza A virus: evaluation in adult seronegative volunteers of $\mathrm{A} / \mathrm{Scot}$ land/840/74 and $\mathrm{A} / \mathrm{Victoria/3/75}$ cold-adapted recombinants derived from the cold-adapted A/Ann Arbor/6/60 strain. Infect. Immun. 23, 253-259 (1979).

27. Neumann, G. et al. Generation of influenza A viruses entirely from cloned cDNAs. Proc. Natl Acad. Sci. USA 96, 9345-9350 (1999).

28. Belshe, R.B. et al. Immunization of infants and young children with live attenuated trivalent cold-recombinant influenza A H1N1, H3N2, and B vaccine. J. Infect. Dis. 165, 727-732 (1992).

29. Gruber, W.C. Children as target for immunization. in Textbook of Influenza (eds Nicholson, K.G., Webster, R.G. \& Hay, A.J.) 435-444 (Blackwell Science, Oxford, UK, 1998).

30. Beyer, W.E., Palache, A.M., de Jong, J.C. \& Osterhaus, A.D. Cold-adapted live influenza vaccine versus inactivated vaccine: systemic vaccine reactions, local and systemic antibody response, and vaccine efficacy. A meta-analysis. Vaccine $\mathbf{2 0}$ 1340-1353 (2002).

31. Russell, S.M. \& Liew, F.Y. T cells primed by influenza virion internal components can cooperate in the antibody response to haemagglutinin. Nature 280, 147-148 (1979).

32. Scherle, P.A. \& Gerhard, W. Differential ability of B cells specific for external vs internal influenza virus proteins to respond to help from influenza virus-specific T-cell clones in vivo. Proc. Natl Acad. Sci. USA 85, 4446-4450 (1988).

33. Webster, R.G. \& Askonas, B.A. Cross-protection and cross-reactive cytotoxic $T$ cells induced by influenza virus vaccines in mice. Eur. J. Immunol. 10, 396-401 (1980).

34. O'Neill, E., Krauss, S.L., Riberdy, J.M., Webster, R.G. \& Woodland, D.L. Heterologous protection against lethal A/HongKong/156/97 (H5N1) influenza virus infection in C57BL/6 mice. J. Gen. Virol. 81, 2689-2696 (2000).

35. Webster, R.G., Bean, W.J., Gorman, O.T., Chambers, T.M. \& Kawaoka, Y. Evolution and ecology of influenza A viruses. Microbiol. Rev. 56, 152-179 (1992). 
TECHNICAL REPORTS

36. Scheiblauer, H., Kendal, A.P. \& Rott, R. Pathogenicity of influenza A/Seal/ Mass/1/80 virus mutants for mammalian species. Arch. Virol. 140, 341-348 (1995).

37. Stech, J., Xiong, X., Scholtissek, C. \& Webster, R.G. Independence of evolutionary and mutational rates after transmission of avian influenza viruses to swine. J. Virol. 73, 1878-1884 (1999).
38. Takada, A., Shimizu, Y. \& Kida, H. Protection of mice against Aujeszky's disease virus infection by intranasal vaccination with inactivated virus. J. Vet. Med. Sci. 56, 633-637 (1994).

39. Kendal, A.P., Pereira, M.S. \& Skehel, J.J. Concepts and procedures for laboratory-based influenza surveillance. Centers for Disease Control, Atlanta, Georgia B17-35, 17-35 (1982). 